\title{
Communication
}

\section{Optimising Digital Health Platforms in Saudi Arabia: Determi- nants from the COVID-19 Pandemic Experience}

\author{
Saeed M Alghamdi 1,2, Abdullah S Alsulayyim ${ }^{3,2}$, Jaber S Alqahtani ${ }^{4,5}$ and Abdulelah M Aldhahir ${ }^{3}$
}

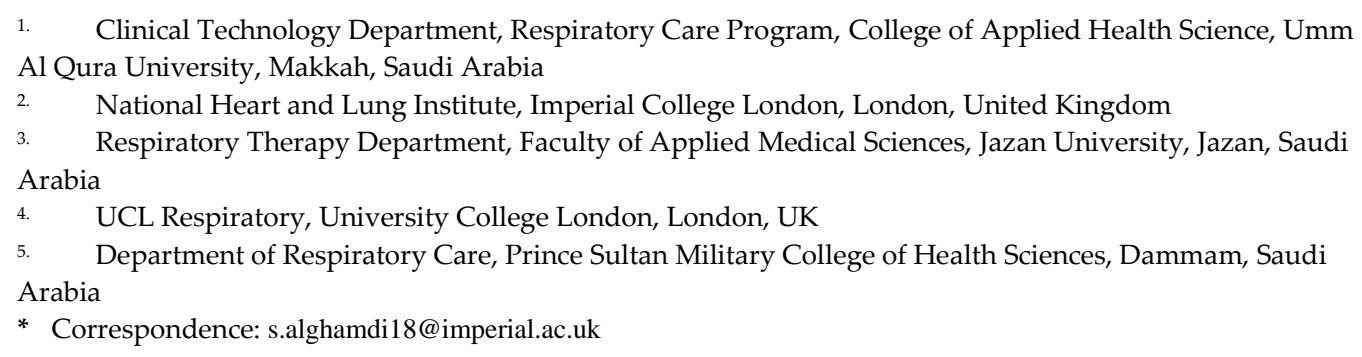

\begin{abstract}
COVID-19 poses a significant burden to healthcare systems. Healthcare organisations with a better health innovation infrastructure have faced a reduced burden and achieved success in curbing COVID-19. In Saudi Arabia, digital technologies have played a vital role in fighting SARS$\mathrm{CoV}-2$ transmission. In this paper, we aimed to summarise the experience of optimising digital health technologies in Saudi Arabia as well as discuss capabilities and opportunities during and beyond the COVID-19 pandemic. A literature review was conducted up to September 2021 to retrieve peer-reviewed articles that critique the use of digital health technology platforms (DHTPs) in Saudi Arabia during the COVID-19 outbreak. A small but significant body of literature examined the digital response to the COVID-19 pandemic in Saudi Arabia. Health officials succeeded in optimising and maintaining a strategy to mitigate the spread of the virus via different digital technologies, such as mobile health applications, artificial intelligence, and machine learning. The quick digital response in Saudi Arabia was facilitated by governmental support and considering users and technology determinants. Future research must concentrate on establishing and updating the guidelines for using DHTPs.
\end{abstract}

Keywords: digital health; health innovation; COVID-19

\section{Introduction}

Coronavirus (COVID-19) has already infected more than 186 nations and claimed thousands of lives, incapacitating even more. Responses to the pandemic have varied dramatically, and the demand on healthcare professionals has increased [1-3].

Around the world, healthcare officials are constantly reviewing appropriate public measures to prevent the spread of COVID-19. For example, minimising physical contact, quarantine, social distancing, and wearing masks have been implemented as measures to keep people safe from infection, especially vulnerable people with chronic conditions. [4] These public health measures have increasingly encouraged the use of technology to support daily activities and healthcare delivery [4]. 
In Saudi Arabia, the Ministry of Health $(\mathrm{MOH})$ recently established a strategy to mitigate COVID-19. The $\mathrm{MOH}$ strategy includes using Digital Health Technology Platforms (DHTPs) to provide long-distance follow-up and control COVID-19 outbreaks [5]. Different terms express the application of DHTPs in healthcare delivery, and these are outlined in Table 1. The aim of this paper is to summarise the experience of using digital health technologies in Saudi Arabia and to discuss capabilities and opportunities during and beyond the COVID-19 pandemic.

Table 1. This table outlines the application of digital health technology platforms (DHTPs) during the COVID-19 pandemic.

\begin{tabular}{|l|l|}
\hline DHTP terminology & Application during COVID-19 outbreak \\
\hline $\begin{array}{l}\text { Digital Health Technology } \\
\text { Platforms (DHTPs) }\end{array}$ & $\begin{array}{l}\text { Using information and communication technologies to } \\
\text { provide distance health services for people as well as } \\
\text { support decision-making processes for healthcare } \\
\text { professionals [6]. }\end{array}$ \\
\hline Artificial Intelligence (AI) & $\begin{array}{l}\text { Using machines to screen people in large public places, } \\
\text { provide fast diagnosis, and detect infected people with } \\
\text { fever [7, 8]. }\end{array}$ \\
\hline Machine Learning (ML) & $\begin{array}{l}\text { Using mathematical algorithms to predict infected } \\
\text { cases, mortality, and vaccination [9, 10]. }\end{array}$ \\
\hline Mobile health applications & $\begin{array}{l}\text { Using mobile apps to track daily symptoms of infected } \\
\text { and non-infected people as well as book appointments } \\
\text { and vaccinations [11]. }\end{array}$ \\
\hline
\end{tabular}

\section{Methods}

A literature search was conducted in English up to September 2021 to retrieve peer-reviewed articles that critique the experience of using DHTPs in Saudi Arabia in response to the COVID-19 outbreak. We also considered any published data, press briefings, news, and announcements by the $\mathrm{MOH}$ in Saudi Arabia. The findings were synthesised in narrative form.

\section{Results}

A small but significant body of literature examined the digital response to the COVID-19 pandemic in Saudi Arabia. Figure 1 visualises the current DHTPs used during the COVID19 outbreak in Saudi Arabia. The strategy was to implement e-health and optimise DHTPs to control the spread of COVID-19 in Saudi Arabia.

\section{Digital response in Saudi Arabia}

Globally, there was a strong drive to implement and accelerate DHTPs. It was led by governments and health authorities to mitigate the spread of the COVID-19. Evidence continues to emerge that using digital health strategies to provide and manage health services is a significant factor in stopping or controlling SARS-CoV-2 transmission. The use of DHTPs has also demonstrated a positive impact on reducing the pressure on healthcare systems and facilitated controlling COVID-19 [12, 13]. 
In Saudi Arabia, the $\mathrm{MOH}$ has succeeded in optimising and maintaining a strategy to mitigate the spread of the virus via different digital health technologies [14]. This strategy includes screening suspected patients, providing daily reports about symptoms, tracing confirmed COVID-19 patients and maintaining social distancing via advanced technology, such as mobile health applications, artificial intelligence, and machine learning (Figure 1).

The development of DHTPs to improve the accessibility of healthcare services across the kingdom was already in place following the Saudi Vision 2030 plan, which stressed the importance of adopting and developing a national telehealth network [15-17].

Optimising DHTPs requires previous experience in the cycle of knowledge translations of health innovations and digital health technologies [16, 18]. This experience had been reported in Saudi Arabia through previous studies, which described the potential and acceptability of DHTPs among the Saudi population. The evidence showed positive results, considering the challenges and limited perspective of clinicians, patients, policymakers, and stakeholders [3, 19-24]. Previous national surveys in Saudi Arabia demonstrated that the DHTPs for COVID-19 had the advantage of wide access, user-friendly interface, ease of use, and high acceptance rate [21-26].

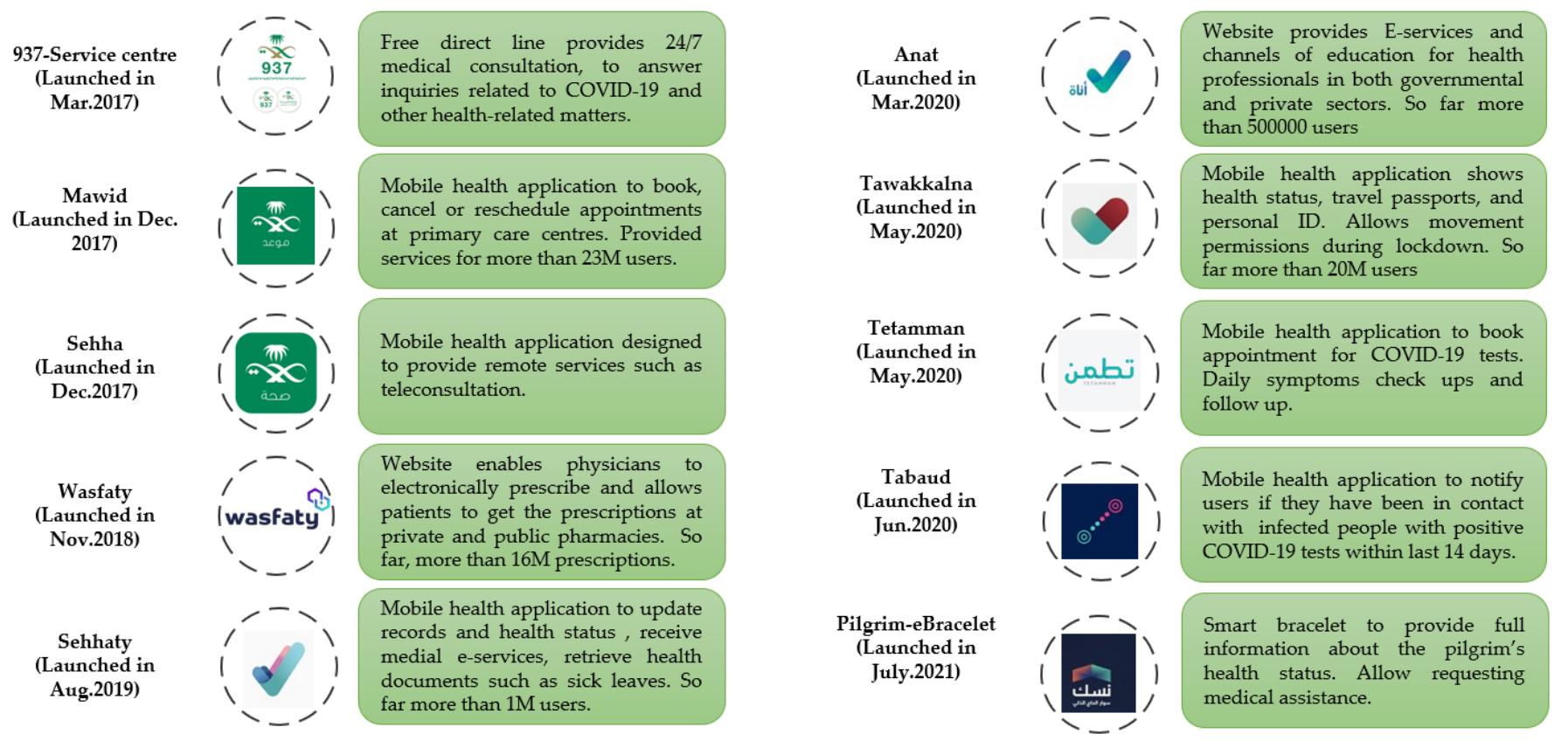

Figure 1. The timeline of optimising DHTPs in Saudi Arabia. Different DHTP applications were launched before, during, and after the COVID-19 pandemic.

\section{Determinants for optimising DHTPs}

In response to the COVID-19 crisis, a multidisciplinary Saudi team from different sectors, including commercial partners, joined forces to overcome these challenges and make the DHTPs easily accessible to citizens and non-citizens in the kingdom of Saudi Arabia.

Strong financial and logistic support from the MOH facilitated DHTPs within a short period of time. According to the $\mathrm{MOH}$ digital transformation report (covering the period 2019-2021), there were more than 23 million registered users on DHTPs such as Mawid, Sehha, Sehhaty, Tawakkalna, Tetamman, and Tabaud.[27] During the pandemic, it was obvious that many outpatient clinics in primary and tertiary hospitals in Saudi Arabia incorporated DHTPs, which facilitated monitoring, managing, and delivering non-urgent medical care in an practical way, thereby maintaining public measures, such as self-isolating and social distancing [28]. (Figure 2) 
The MOH in Saudi Arabia developed and introduced additional features for current DHTPs to cope with the COVID-19 pandemic. These were effectively incorporated into the healthcare system. Not only did DHTPs provide benefits but trained human support via free direct line 937, both technical and healthcare providers, was also available 24/7 and people were able to access services from any location in Saudi Arabia [3, 29]. The strategy was not to replace the human factor with technology; it was to support the technology with a greater human factor to optimise its effectiveness in fighting COVID19.

Data availability was an essential determinant for optimising the DHTPs during COVID-19. Since patient zero was discovered, non-confidential data of COVID-19 have been available and free to use to prompt health innovations and digital health technology applications. Many researchers have used the data to model the future of the pandemic or to predict new cases and deaths in the upcoming months [30-34]. Data accessibility has facilitated building models to predict the future of the pandemic in Saudi Arabia and has helped decision-makers to set plans and strategies to fight COVID-19 [35, 36].

The technical determinant of optimising DHTPs in Saudi Arabia was the robust infrastructure of both networks and technologies [37]. The MOH in Saudi Arabia effectively encouraged people to use DHTPs to receive care rather than visiting primary care clinics during the COVID-19 pandemic [3, 16]. DHTPs were central to the role of primary health services to help with triage and mitigating the spread of COVID-19 [1, 2, 38, 39].

Internet connectivity is widely available to the population in Saudi Arabia, which has supported the healthcare system to continually provide high-quality care for all patients in the private and public sectors [28]. Saudi Arabia is identfied as one of the countries in the Middle East where more than $90 \%$ of its population have internet connections [40]. Big telecom companies in Saudi Arabia, such as Saudi Telecom Company (STC), Zein, and Mobily, announced free data use for the DHTPs during the pandemic, which ultimately facilitated high usage and smooth usability of the technologies [3].

" Years 2019 and $2020 \quad$ Year 2021

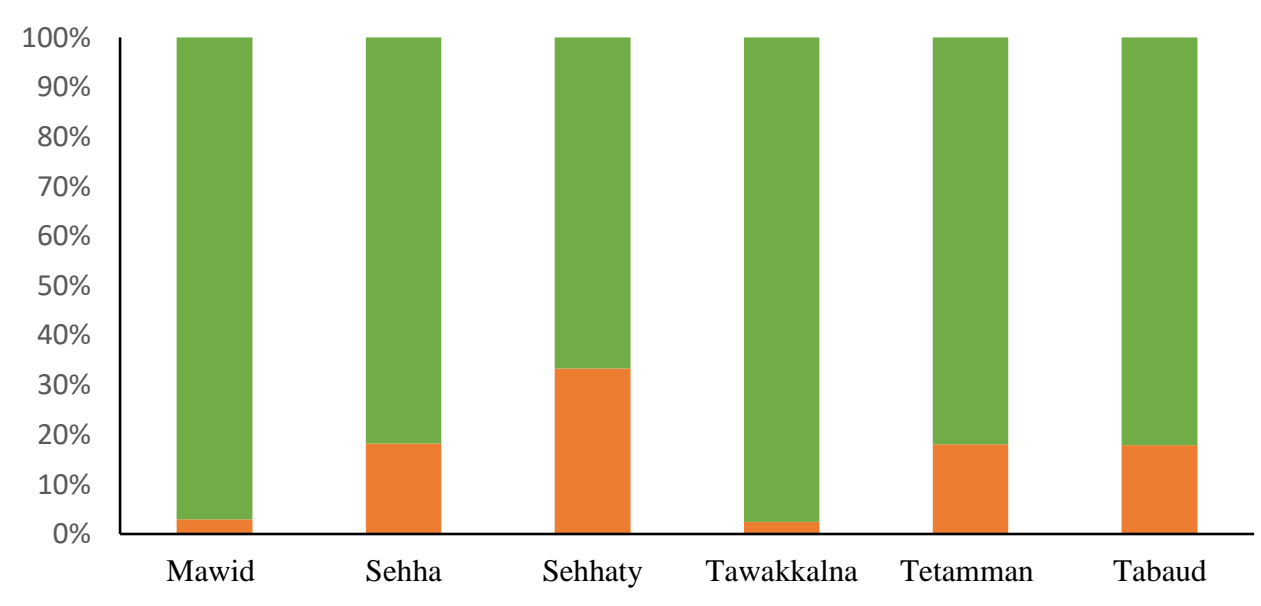

Figure 2. Increase the number of registered users of DHTPs over the period from 2019 to 2021. Data presented as proportions. [16, 27] 


\section{DHTPs and Hajj season}

A success story must be told about the Hajj season in 2020. With the help of DHPTs, the authorities in Saudi Arabia transformed the Hajj season from a super spreading event and COVID-19 variant farm into a safe and healthy season with zero COVID-19 cases $[14,41]$.

Prior to the COVID-19 pandemic, health authorities in Saudi Arabia were prepared for any potential spread of infectious diseases associated with mass gatherings (e.g. Hajj season) [42]. The coinciding of COVID-19 with the Hajj season is an extra indication of the insights and benefits that DHTPs can provide, such as predicting the progression and the effects of COVID-19 during the Hajj season via mathematical modelling and machine learning. A recent study has examined agent-based modelling in curbing COVID-19 spread during the Hajj season. The findings showed that control measures, such as buffers and face masks, are simple but have a significant impact on controlling infection, especially during the Hajj season. This mathematical modelling provides robust information to support decision-making about public measures [14, 43].

One of the advanced health innovations launched during the Hajj was the "Pilgrim eBracelet" to monitor Hajj performers, screen their daily symptoms, and monitor the mass gathering. The smart bracelet is connected to the internet to provide several services, such as monitoring the Hajj performers' health condition and any change in exposure to COVID-19 cases. The smart bracelet also provides information about the heart rate and oxygen saturation. Moreover, the bracelet allows the Hajj performers to seek assistance in case of an emergency. Only 5000 Pilgrim eBracelets were distributed to pilgrims as a trial version. Outcomes were surprising and the season ended with zero infection cases. This suggests that DHTPs may help to design a safe and secure environment to perform Hajj without any unexpected cases of infection [14, 44, 45].

\section{Conclusion}

Health officials in Saudi Arabia succeeded in optimising and maintaining a strategy to mitigate the spread of the virus via different digital technologies, such as mobile health applications, artificial intelligence, and machine learning. The quick digital response in Saudi Arabia was facilitated by governmental support and considering users and technology determinants. Considering all of this success, we still need further work to sustain the use of DHTPs and explore the experience of artificial intelligence in guiding clinical practice. We also need to increase the interconnected network between primary and tertiary health centres to share electronic medical records with other healthcare centres for a better interpretation and management strategy.

\section{Reference}

1. Ministry of Health (Saudi Arabia) : National E- Health Strategy. 2021 Available from: https://www.moh.gov.sa/en/Ministry/nehs.

2. Ministry of Health (Saudi Arabia) : COVID-19 Daily briefing. 20212020 Aug 10; Available from: https://www.moh.gov.sa/en/Ministry/MediaCenter.

3. Hassounah, M., H. Raheel, and M. Alhefzi, Digital response during the COVID-19 pandemic in Saudi Arabia. Journal of Medical Internet Research, 2020. 22(9): p. e19338.

4. Monaghesh, E. and A. Hajizadeh, The role of telehealth during COVID-19 outbreak: a systematic review based on current evidence. BMC Public Health, 2020. 20(1): p. 1-9.

5. Khan, A., et al., COVID-19 in Saudi Arabia: the national health response. East Mediterr Health J, 2021.

6. Hollander, J.E. and B.G. Carr, Virtually perfect? Telemedicine for COVID-19. New England Journal of Medicine, 2020. 382(18): p. 1679-1681. 
7. Vaishya, R., et al., Artificial Intelligence (Al) applications for COVID-19 pandemic. Diabetes \& Metabolic Syndrome: Clinical Research \& Reviews, 2020. 14(4): p. 337-339.

8. Alassaf, N., et al., Evaluation of Official Healthcare Informatics Applications in Saudi Arabia and their Role in Addressing COVID-19 Pandemic. Healthcare informatics research, 2021. 27(3): p. 255.

9. Kushwaha, S., et al., Significant applications of machine learning for COVID-19 pandemic. Journal of Industrial Integration and Management, 2020. 5(4).

10. Omran, N.F., et al., Applying Deep Learning Methods on Time-Series Data for Forecasting COVID-19 in Egypt, Kuwait, and Saudi Arabia. Complexity, 2021. 2021.

11. Adeniyi, E.A., et al., Mobile health application and COVID-19: Opportunities and challenges. Journal of Critical Reviews, 2020. 7(15): p. 3481-3488.

12. Fagherazzi, G., et al., Digital health strategies to fight COVID-19 worldwide: challenges, recommendations, and a call for papers. Journal of Medical Internet Research, 2020. 22(6): p. e19284.

13. Gunasekeran, D.V., et al., Applications of digital health for public health responses to COVID-19: A systematic scoping review of artificial intelligence, telehealth and related technologies. NPJ digital medicine, 2021. 4(1): p. 1-6.

14. Jokhdar, H., et al., COVID-19 mitigation plans during Hajj 2020: a success story of zero cases. Health security, 2021. 19(2): p. 133-139.

15. Ministry Of Health (Saudi Arabia) : National E- Health Strategy. 20212021 April 23 [cited 2021 July 19 ]; Available from: https://www.moh.gov.sa/en/Ministry/nehs.

16. Alghamdi, S.M., J.S. Alqahtani, and A.M. Aldhahir, Current status of telehealth in Saudi Arabia during COVID19. Journal of family \& community medicine, 2020. 27(3): p. 208.

17. Aldhahir AM, A.J., Althobiani MA, Alghamdi SM, Alanazi AF, Alnaim N, Alqarni AA, Alwafi H Current Knowledge, Satisfaction, and Use of E-health Mobile Application (Seha) among the General Population of Saudi Arabia: A National Survey. JMIR mHealth and uHealth, 2021. DOI: http://doi.org/10.2196/preprints.33326

18. Gagnon, M.-P., et al., A systematic review of instruments to assess organizational readiness for knowledge translation in health care. PloS one, 2014. 9(12): p. e114338.

19. Al-Dossary, R., et al., Awareness, attitudes, prevention, and perceptions of COVID-19 outbreak among nurses in Saudi Arabia. International journal of environmental research and public health, 2020. 17(21): p. 8269.

20. Blandford, A., et al., Opportunities and challenges for telehealth within, and beyond, a pandemic. The Lancet Global Health, 2020. 8(11): p. e1364-e1365.

21. ALOmari, M.O. and J. Jenkins, Exploring the Attitudes of Patients towards using the Seha Application (Telehealth) in Saudi Arabia during the Coronavirus Epidemic. ABC Journal of Advanced Research, 2021. 10(1): p. 9-22.

22. Alhamam, N.M., et al., Telemedicine for Musculoskeletal Care During the COVID-19 Pandemic: Evaluating Readiness of Saudi Citizens. Cureus, 2021. 13(2).

23. Alharbi, A., J. Alzuwaed, and H. Qasem, Evaluation of e-health (Seha) application: a cross-sectional study in Saudi Arabia. BMC medical informatics and decision making, 2021. 21(1): p. 1-9.

24. Robert, A.A., A. Al Saeed, and M.A. Al Dawish, COVID-19 among people with diabetes mellitus in Saudi Arabia: Current situation and new perspectives. Diabetes \& Metabolic Syndrome: Clinical Research \& Reviews, 2021: p. 102231.

25. Mubaraki, A.A., et al., Advantages and disadvantages of telemedicine during the COVID-19 pandemic era among physicians in Taif, Saudi Arabia. Saudi medical journal, 2021. 42(1): p. 110. 
26. Nasser, A.A., et al., Measuring the Patients' Satisfaction About Telemedicine Used in Saudi Arabia During COVID-19 Pandemic. Cureus, 2021. 13(2).

27. National Digital Transformation Report Annual report 2021 September 16; First Edition:[113]. Available from: https://ndu.gov.sa/en/.

28. Alhudhaif, A. Role of Technology in Managing COVID-19: A Case of Saudi Arabia. in 2021 8th International Conference on Computing for Sustainable Global Development (INDIACom). 2021. IEEE.

29. Daem Service : Saudi Commission for Health Specialties 2021 [cited 2021 July 30 ]; Available from: https://www.scfhs.org.sa/en/eservices/trainees/Pages/TrainingGuidanceServiceDesc.aspx.

30. Bachar, M., M.A. Khamsi, and M. Bounkhel, A mathematical model for the spread of COVID-19 and control mechanisms in Saudi Arabia. Advances in Difference Equations, 2021. 2021(1): p. 1-18.

31. Sharma, S.K. and S.S. Ahmed, IoT-based analysis for controlling \& spreading prediction of COVID-19 in Saudi Arabia. Soft Computing, 2021: p. 1-13.

32. Mohamed, I.A., et al., A new model for epidemic prediction: COVID-19 in kingdom saudi arabia case study. Materials Today: Proceedings, 2021.

33. Komies, S., et al., COVID-19 Outcomes in Saudi Arabia and the UK: A Tale of Two Kingdoms. medRxiv, 2020.

34. Alshammari, F.S., A mathematical model to investigate the transmission of COVID-19 in the Kingdom of Saudi Arabia. Computational and Mathematical Methods in Medicine, 2020. 2020.

35. Alyami, M.H., et al., Epidemiology of COVID-19 in the Kingdom of Saudi Arabia: an ecological study. Frontiers in Public Health, 2020. 8.

36. Adly, H.M., et al., Correlation of COVID-19 Pandemic with Healthcare System Response and Prevention Measures in Saudi Arabia. International Journal of Environmental Research and Public Health, 2020. 17(18): p. 6666.

37. Algaissi, A.A., et al., Preparedness and response to COVID-19 in Saudi Arabia: Building on MERS experience. Journal of infection and public health, 2020. 13(6): p. 834-838.

38. Saudi Center for Disease Prevention and Control : (Covid-19) Disease Interactive Dashboard 20202020 Aug 10; Available from: https://covid19.cdc.gov.sa/daily-updates.

39. Alqahtani, J.S., et al., Clinical practice and barriers of ventilatory support management in COVID-19 patients in Saudi Arabia: A survey of respiratory therapists. Saudi Journal of Medicine and Medical Sciences, 2021. 9(3): p. 223.

40. Saquib, J., Internet addiction among Saudi Arabian youth. International journal of health sciences, 2020. 14(2): p. 1.

41. National Digital Transformation Report. Samrt Hajj 2021 [cited 2021 September 15]; Available from: https://ndu.gov.sa/report/Haji-report.pdf.

42. Memish, Z.A., et al., Hajj: infectious disease surveillance and control. The Lancet, 2014. 383(9934): p. 20732082.

43. Al-Shaery, A.M., et al., Agent-Based Modeling of the Hajj Rituals with the Possible Spread of COVID-19. Sustainability, 2021. 13(12): p. 6923.

44. Alshalani, H., N. Alnaghaimshi, and S. Eljack. ICT System for Crowd Management: Hajj as a Case Study. in 2020 International Conference on Computing and Information Technology (ICCIT-1441). 2020. IEEE.

45. Shambour, M.K. and A. Gutub, Progress of IoT Research Technologies and Applications Serving Hajj and Umrah. Arabian Journal for Science and Engineering, 2021: p. 1-21. 